\title{
Phase morphology and crystallinity of poly(vinylidene fluoride)/ poly(ethylene oxide) piezoelectric blend membranes
}

\author{
M.N. Tamaño-Machiavello ${ }^{1 *}$, C. M. Costa $^{2}$, J. Molina-Mateo ${ }^{1}$, C. Torregrosa- \\ Cabanilles $^{1}$, J.M Meseguer-Dueñas ${ }^{1,3}$, S. N. Kalkura ${ }^{4}$, S. Lanceros-Méndez², R. Sabater \\ i Serra ${ }^{1}$, J. L. Gómez Ribelles ${ }^{1,3}$ \\ 1) Center for Biomaterials and Tissue Engineering, CBIT, Universitat Politècnica de València, \\ 46022 Valencia, Spain \\ 2) Centro/Departamento de Física, Universidade do Minho, 4710-057 Braga, Portugal \\ 3) Networking Research Center on Bioengineering, Biomaterials and Nanomedicine \\ (CIBER-BBN), Valencia, Spain \\ 4) Crystal Growth Centre, Anna University, Chennai, 600025 INDIA
}

(*) Corresponding author:

Name: María Noel Tamaño Machiavello

e-mail: noeltm@gmail.com

\begin{abstract}
:
Polymer blends based on poly(vinylidene fluoride), PVDF and poly(ethylene oxide), PEO, with varying compositions have been prepared by solvent casting, the polymer blend films being obtained from solutions in dimethyl formamide at $70^{\circ} \mathrm{C}$. Under these conditions PVDF crystallizes from solution while PEO remains in the molten state. Then, PEO crystallizes from the melt confined by PVDF crystalls during cooling to room temperature. PVDF crystallized from DMF solutions adopt predominantly the electroactive $\beta$-phase (85\%). Nevertheless when PEO is introduced in the polymer blend the $\beta$-phase content decreases slightly to $70 \%$. The piezoelectric coefficient $\left(d_{33}\right)$ in pristine $\mathrm{PVDF}$ is $-5 \mathrm{pC} / \mathrm{N}$ and decreases with increasing PEO content in the PVDF/PEO blends. Blend morphology, observed by electron and atomic force microscopy, shows the confinement of PEO between the already formed PVDF crystals. On the other hand the sample contraction when PEO is extracted from the blend with water (which is not a solvent for PVDF) allows proving the co-continuity of both phases in the blend. PEO crystallization kinetics have been characterized by DSC both in isothermal and cooling scans experiments showing important differences in crystalline fraction and crystallization rate with sample composition.
\end{abstract}

Keywords: PVDF, PEO, polymer blends, piezoelectric polymer 


\section{Introduction}

In recent years there has been a growing interest in the field of membrane technology, more specifically, in polymer porous membranes with the objective to promote or improve its performance in applications such as water purification [1], reverse osmosis [2], biomedical [3] and energy applications [4-6], etc. Polymer porous membranes show many advantages in comparison of other membrane types, such as metal and liquid membranes. In particular: they can show a high variety of the pore sizes and degree of porosity, various membrane shapes (flat sheet, tubular, etc) and can be chemically resistant, among others [7-9].

Different methods have been developed to generate a well-controlled pore structure and pore interconnectivity. Solvent casting with phase inversions is one of the most used methods [9-12] but many other have been proposed.

The most used polymer materials for porous membrane development for different applications are polycarbonate (PC) [13], poly(vinylidene fluoride) (PVDF) [14], polytetrafluoroethylene (PTFE) [15], polypropylene (PP) [16], polyamide [17] and cellulose-ester [18]. Taking into account the excellent properties (high mechanical strength and chemical stability) of the fluoropolymers, PVDF has been used in porous membranes fabrication. PVDF is known for its electroactive properties (piezo, pyro and ferroelectric properties) $[19,20]$. On the other hand, PVDF is a hydrophobous biocompatible and non-biodegradable material, suitable for cell culture applications.

We propose in this work the combination of PVDF with PEO in a non-porous membrane in which a hydrophobous and a hdrophilic phases are co-continuous. This structure will allow water sorption and diffussion when the membrane is used as a cell culture support allowing cell attachment to PVDF domains but at the same time allowing the diffusion of water soluble proteins or growth factors through the substrate for cell signalling [21-23]. The ability of absorbing polar solvents is crucial in the use of these membranes as litium ion battery separator [24, 25], or in microfiltration $[26,27]$ 
PVDF is a semi-crystalline polymer that can crystallize in four crystalline phases known as: $\alpha, \beta, \gamma$ and $\delta$ and depending on the temperature and processing conditions [20, 2830]. The most important phase of PVDF for technological applications is the $\beta$-phase, as it exhibits piezoelectric, pyroelectric and ferroelectric, properties. The $\alpha$-phase is the most stable one from a thermodynamic point of view when the material is directly obtained from the melt [26-28]. Porous $\beta$-PVDF samples can be obtained directly from solution crystallization at temperatures below $70^{\circ} \mathrm{C}$ [31]. Mechanical stretching applied to an $\alpha$-phase PVDF film at temperatures below $100^{\circ} \mathrm{C}$ and with stretch ratio higher than or equal to 3 transforms it to a non-porous $\beta$-phase film. Different morphologies and microstructures were obtained for PVDF microporous membranes by crystallizing at different temperatures (thermal induced phase separation, TIPS) [31, 32]. TIPS allows controlling both porosity and pore size $[32,33]$. The crystalline phase, dielectric and thermal properties of PVDF membranes depend of the solvent evaporation temperature that influences the solvent evaporation rate below of the melting temperature $[34,35]$. For biomedical applications, the influence of the polarization state of non-porous electroactive poly(vinylidene fluoride), PVDF, on the biological response of cells cultured under static and dynamic conditions has been addressed [36, 37]. It was observed that positively charged $\beta$-PVDF films promote higher osteoblast adhesion and proliferation, which is higher under dynamic conditions on poled samples, showing that the surface charge under mechanical stimulation improves the osteoblast growth.

Poly(ethylene oxide), PEO is a hydrophilic, biocompatible polymer that has been intensively used in biomedical application [38]. Polymer blends of PVDF and PEO have been developed to improve the pore configuration, such as pore size, porosity and pore connectivity of PVDF-based microporous membranes [39, 40]. This polymer blend has been proven to be suitable for polymer electrolyte applications [40].

Taking into account the properties of both PVDF and PEO, the main goal of this work is the preparation of new polymer blends based on these polymers in order to properly tune morphological features, with suitable piezoelectric and thermal properties for biomedical and energy applications. The correlation between the phase morphology and electroactive phase of PVDF is extremely important for these applications. A new membrane preparation method has been developed in which PVDF crystallizes from the solution in DMF while PEO polymer crystallizes from the melt confined between PVDF crystals. 


\section{Experimental details}

\subsection{Blend preparation}

$\operatorname{PVDF}\left(\mathrm{M}_{\mathrm{w}} 700000 \mathrm{~g} / \mathrm{mol}\right)$ and PEO (M $\left.100000 \mathrm{~g} / \mathrm{mol}\right)$ were acquired from Solvay and Polysciences, respectively. PVDF/PEO blends were prepared with compositions of 100/0, 70/30, 50/50 weight ratio. Blends were prepared by dissolving the adequate amounts of both polymers in N,N-dimethylformamide (DMF) at a 15/85 w/v polymer/ solvent ratio. The polymers were dissolved at $80{ }^{\circ} \mathrm{C}$ during $48 \mathrm{~h}$ with the help of a magnetic stirrer in glass vessel with outer jacket for circulation of water until a homogeneous and transparent solution could be observed. The solutions were deposited in Petri's capsules and the solvent was allowed to evaporate at $70^{\circ} \mathrm{C}$ for two hours. Finally, complete removal of the solvent was achieved in vacuum for another 3 hours at $70{ }^{\circ} \mathrm{C}$.

\subsection{Removal of PEO}

As PEO is a hydrophilic polymer, it can be removed by immersing in water. The membranes were cut into cylinders of $5 \mathrm{~mm}$ in diameter. The cylinders were immersed in water and stirred during up to 7 days, while the water was changed every day. After 7 days, the samples were dried in open air and subsequently dried in vacuum at $40{ }^{\circ} \mathrm{C}$ for one day. The mass, thickness and diameter of the samples were measured before and after washing in water.

The percentage of removal of PEO was measured at various times and calculated through the following equation 1 :

$$
\% \text { PEO removal }=\left(\frac{W_{0}-W_{i}}{W_{0}}\right) \times 100
$$

where $W_{0}$ and $W_{i}$ denote the weight of blend membrane and the weight of membrane after PEO extraction by soaking in water, respectively. 


\subsection{Sample Characterization:}

The microstructure of the blend films were examined in a field emission scanning electron microscope (FESEM), ZEISS Ultra-55, after deposition of a conductive layer of sputtered platinum. AFM experiments were performed in tapping mode in air, immediately after sample preparation, using a Multimode AFM equipped with NanoScope IIIa controller from Veeco (Manchester, UK), at ambient conditions. Sicantilevers with a constant force of $2.8 \mathrm{~N} / \mathrm{m}$ and a resonance frequency of $75 \mathrm{kHz}$ were used. All the samples were characterized using a set-point amplitude ratio of around 0.7 The NanoScope 5.30r2 software version was used for the simultaneous recording of the height, phase, and amplitude magnitudes of the images.

The PVDF crystalline phase was identified by Fourier Transformed Infrared spectroscopy (FTIR) at room temperature with a Jasco FTIR-4100 set-up. FTIR spectra were collected in attenuated total reflectance mode (ATR) from 4000 to $600 \mathrm{~cm}^{-1}$ after 32 scans with a resolution of $4 \mathrm{~cm}^{-1}$.

For determination of the piezoelectric coefficient, the PVDF/PEO blends were first poled by corona discharge at a controlled temperature inside a home-made corona chamber with the following parameters, obtained after an optimization process: applied voltage of $10 \mathrm{kV}$ at a constant current of $20 \mu \mathrm{A}$; constant distance of $1.5 \mathrm{~cm}$ between the sample and the tip; poling time $60 \mathrm{~min}$, poling temperature of $60^{\circ} \mathrm{C}$ and then cooled to room temperature under the applied electric field.

Differential scanning calorimetry (DSC) analysis was carried out with a Perkin-Elmer DSC 8000 instrument under a flowing nitrogen atmosphere between 25 and $200{ }^{\circ} \mathrm{C}$ at a heating rate of $20{ }^{\circ} \mathrm{C} \mathrm{min}{ }^{-1}$ for cooling and heating. All samples were measured in $30 \mu \mathrm{L}$ aluminum pans with perforated lids to allow the release and removal of volatiles. 


\section{Results and Discussion}

\subsection{Phase Morphology}

PVDF/PEO blend films were casted from the solution in a good solvent for both components. Solvent casting was performed at $70^{\circ} \mathrm{C}$ to ensure that during the whole process PEO is amorphous. PVDF crystallizes from the solution during solvent evaporation and, it is expected that PEO chains are pushed by the growing PVDF crystals, being finally confined in spaces between PVDF spherulites or in interlamelar spaces mixed with amorphous PVDF chains. Then when the already dry film is cooled to room temperature, PEO crystallizes from the melt since no solvent rests in the sample. As we will see below PEO crystallization rate is highly affected by the presence of PVDF. It is worth note that simultaneous growth of PEO and PVDF spherulites during solvent casting at room temperature yields a brittle and inhomogeneous film with poor adhesion between the tow crystalline phases (results not shown).

In order to characterize the phase morphology in the blend thus obtained, PEO was extracted from the blend film by washing in water, which is a non-solvent for PVDF. Soluble fraction of PEO when immersing the film in water will characterize the amount of PEO which dispersed in isolated domains, while the morphology of the remaining PVDF and contraction will speak about connectivity of PVDF phase in the blend.

After washing the samples for 7 days, the mass loss of the membrane is, as expected, approximately $50 \%$ for the membrane with a PVDF/PEO ratio of 50/50. Thus, it can be said that PEO phase is continuous in the membrane. Nevertheless only around $25 \%$ mass is lost by the 70/30 blend indicating that in this case some PEO is confined within the structure of the PVDF, where water cannot penetrate. These results are shown in figure 1a. Figures $1 \mathrm{~b}$ and $1 \mathrm{c}$ show the thickness, width and volume decrease in the membrane as a function of immersion time. The volume decrease is due to the collapse of the membrane after removal of PEO, forming a more compact material. Taking into account the densities of pure PEO and PVDF 1,130 and 1,78g $\mathrm{cm}^{-3}$ respectively the volume occupied by PEO in 50/50 blend should be $61 \%$. The volume contraction when extracting PEO is $70 \%$ so the collapse of the membrane is confirmed. 
However, in the case of the 70/30 sample, the volume fraction occupied by PEO should be $32 \%$ but volume loss when extracting it is only $25 \%$ supporting again that part of PEO is still in the blend but also that continuity of PVDF crystalline structures hinders sample contraction leaving a proportionally more porous membrane. Interestingly, sample contraction is not isotropous. Samples were in the form of cylinders and thickness reduction in 50/50 sample is $35 \%$ while diameter reduction was $30 \%$ (results not shown), in sample 70/30 thickness reduction was $15 \%$ while diameter one was around 8\% (results not shown). This means that the sample surface contracts less than thickness. On the other hand it is interesting to observe that while PEO rapidly leaves the sample, sample contraction takes longer. In fact after one hour washing in water, $90 \%$ of the PEO that the blend contains initially has been extracted, as shown by the evolution of the sample mass (Figure 1a). Nevertheless, volume only stabilizes after 3 hours showing that the collapse of PVDF structure is governed by a relaxation process
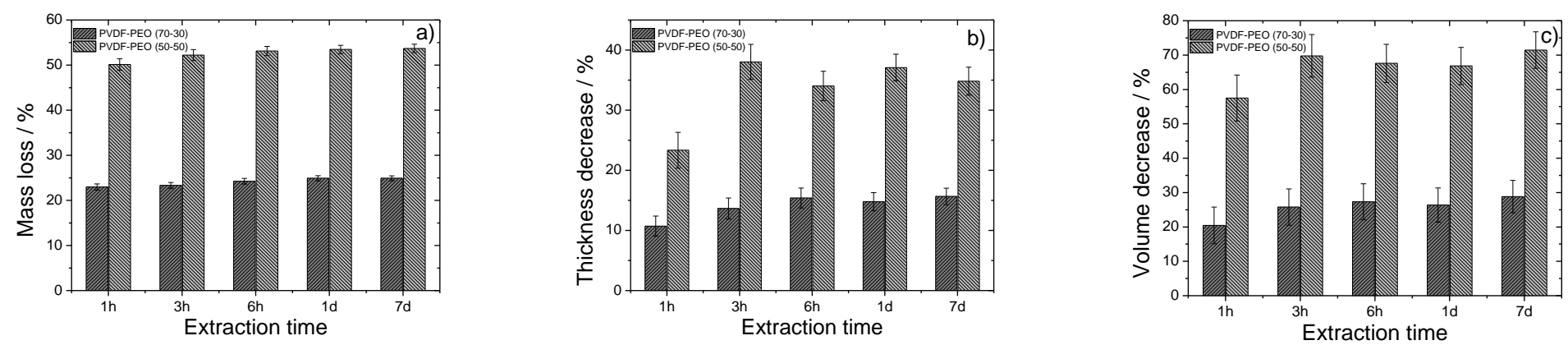

Figure 1 - a) Mass loss, b) thickness decrease and c) volume decrease as a function of PEO extraction for all PVDF-PEO blends as a function of washing time in water.

These results can be understood observing blend morphology before and after PEO extraction. Surface and cross-section FESEM images are shown in figures 2 and 3 for PVDF/PEO 50/50 and 70/30, respectively. Figures $2 \mathrm{a}$ and $2 \mathrm{~b}$ represent the morphology for the pure 70/30 PVDF/PEO blend through the surface and cross-section FESEM images, respectively. 


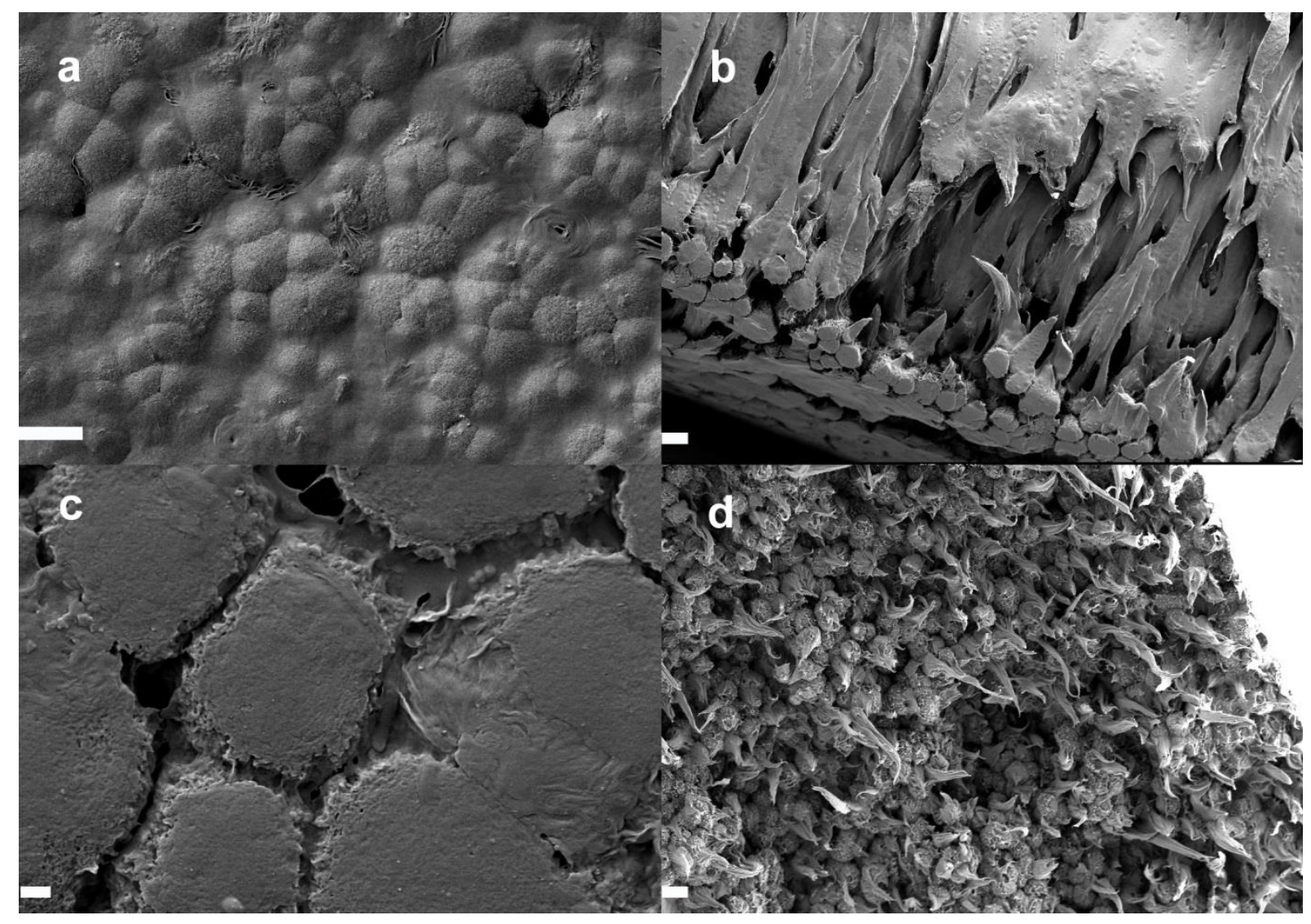

Figure 2- Surface and cross-section FESEM images: a-b) PVDF/PEO (70/30) blends and c-d) after PEO extraction for 7 days. Dimension bar correspond to $10 \mu \mathrm{m}$ in (a) (b) (d) and $1 \mu \mathrm{m}$ in (c)

Phase separation can be observed already in the image of the surface of 70/30 blend (Figure 2a). Structures that seem polymer spherulites are apparent and we will see that they correspond to PVDF when observing the picture after PEO extraction (Figure 2c). The topography shown in the polymer blend must be the result of PEO crystallization on cooling from $70^{\circ} \mathrm{C}$ to room temperature. The formation of PEO crystalline lamellae produces some texture at the surface. In the case of 50/50 sample the blend shows some PVDF circular regions separated by rough regions of semicrystalline PEO (Figure 3a).

It is interesting to observe the huge difference of the cryogenic cross section of the $50 / 50$ and $70 / 30$ blends (Figures $2 b$ and $3 b$ respectively). While in 70/30 blend cryogenic fracture seems to disaggregate PVDF domains, in 50/50 sample cross section is perfectly smooth with no sign of phase separation between PVDF and PEO.

After PEO extraction, the surface in both blends shows circular PVDF aggregates, with quite rough surfaces in the case of 50/50 sample. It seems like if when PVDF 
spherulites grow during DMF evaporation at $70^{\circ} \mathrm{C}$, some $\mathrm{PEO}$ is retained within the semicrystalline PVDF domains. On the other hand, in the cross section, after extracting PEO, PVDF structures appear as $1 \mu \mathrm{m}$ spheres quite well separated from each other. Nevertheless, in 50/50 sample the structure seems to collapse in more extent and, in fact, PVDF domains clearly shown at the surface can be hardly distinguished in the cross-section. These features, which agree with the data of sample contraction, can be explained by the formation of PVDF bridges between the spherulites during crystallization when the amount of PEO is small. Growing crystals adhere to each other stabilizing the PVDF architecture that is kept when PEO is extracted. On the contrary, when the amount of PEO is higher, PVDF crystals grow without contact with each other, dispersed in the PEO matrix and when PEO disappears collapse in larger extent.

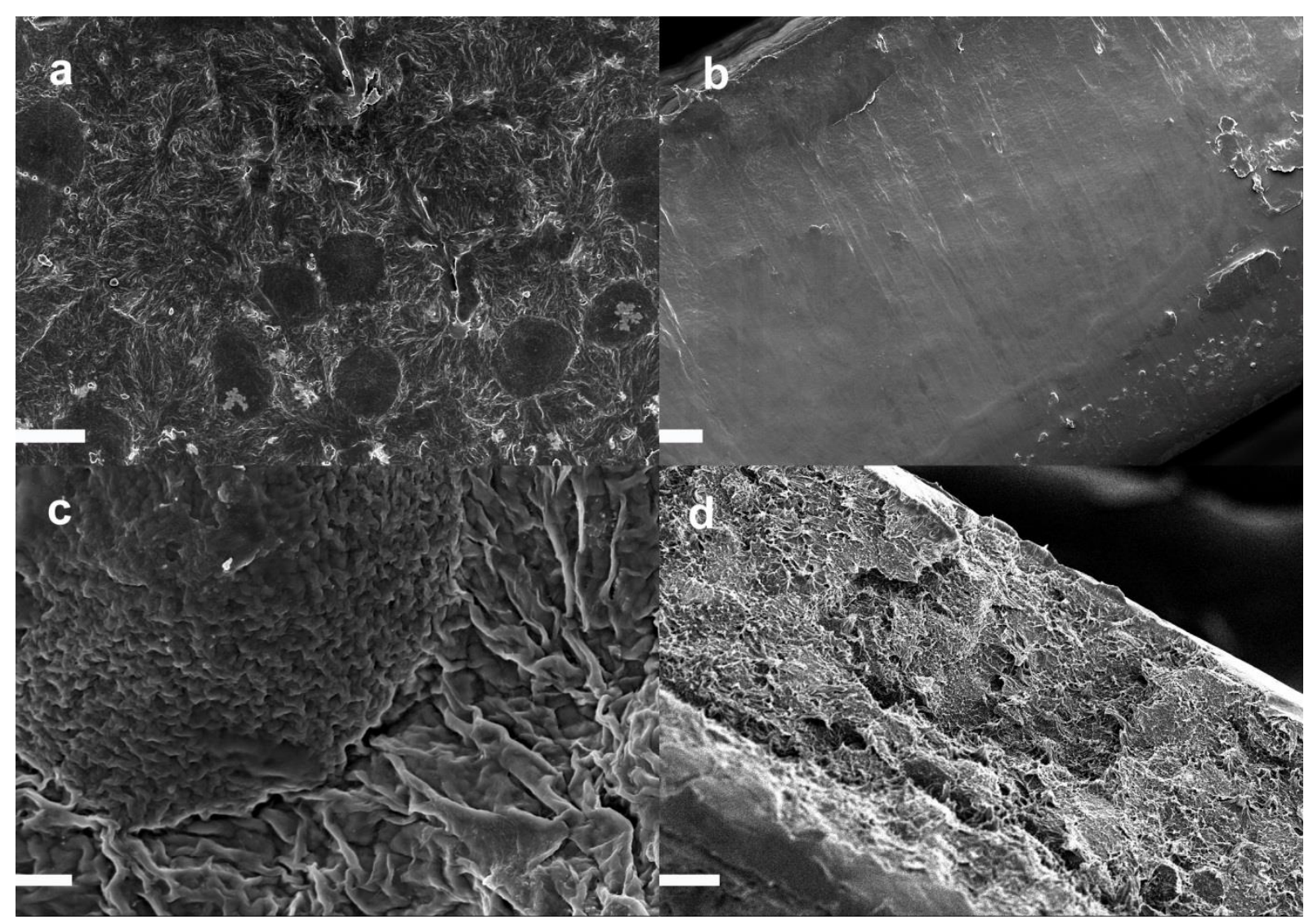

Figure 3 - Surface and cross-section FESEM images: a-b) PVDF/PEO (50/50) blends and c-d) after PEO extraction for 7 days. . Dimension bar correspond to $10 \mu \mathrm{m}$ in (a) (b) (d) and $1 \mu \mathrm{m}$ in (c) 
Surface topography can be observed with more detail with AFM (Figure 4). Tapping mode AFM shows the presence of the two phases at the surface of the sample but without significant height differences between them (Figures $4 \mathrm{a}$ and $4 \mathrm{c}$ ). PEO extraction emphasizes PVDF regions (Figures $4 \mathrm{~b}$ and $4 d$ )

a)

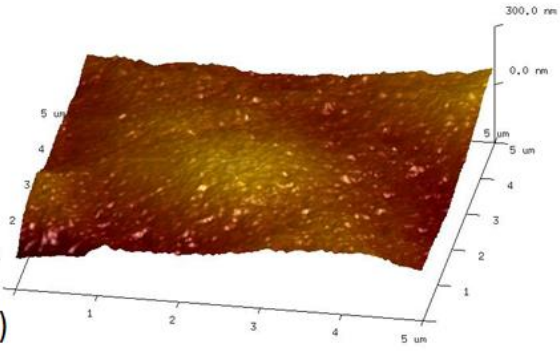

c)

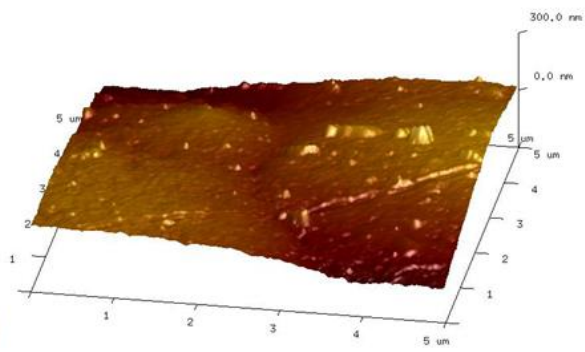

b)
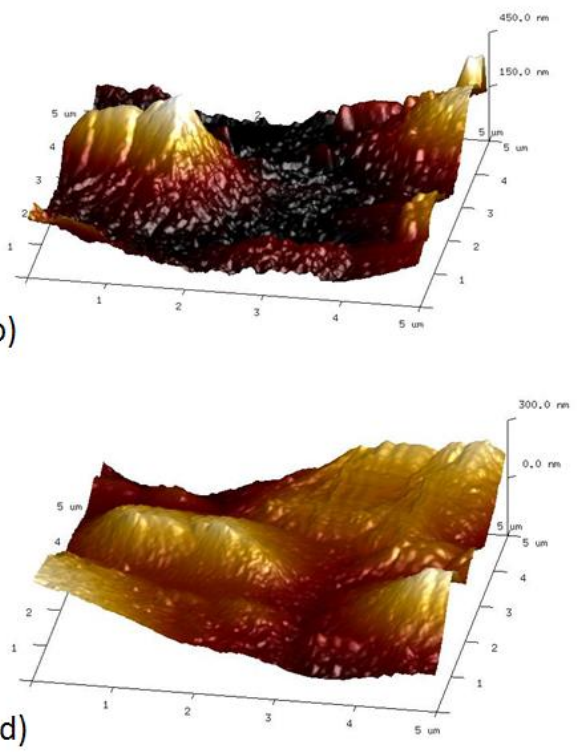

Figure 4: AFM height images of PVDF/PEO blends a) PVDF/PEO (50/50) blends, b) (50/50) after PEO extraction, c) PVDF/PEO (70/30) blends and d) (70/30) after PEO extraction.

\subsection{Infrared spectroscopy and piezoelectric response}

FTIR spectroscopy was used to characterize crystalline phase of PVDF and PEO polymers [30]. The two most relevant crystalline phases of PVDF are the $\alpha$-phase and $\beta$-phase, the specific bands characteristics for $\alpha$-phase being 765, 796, 855 and $976 \mathrm{~cm}^{-1}$ while $\beta$-phase is identified by 840 and $1232 \mathrm{~cm}^{-1}$, bands [30]. On the other hand, the most important specific bands characteristics of PEO are 845-948, 1280, 1343 and 1468 $\mathrm{cm}^{-1}$ corresponding of $\mathrm{CH}_{2}$ rocking, twisting, wagging and scissoring, respectively [41]. FTIR-ATR spectra for PVDF/PEO blends and pristine PEO are shown in figure 5.

For the PVDF pristine sample, the small vibration bands at $760 \mathrm{~cm}^{-1}$, that correspond to $\alpha$ phase crystals and the specific band at $838 \mathrm{~cm}^{-1}$ characteristics of the $\beta$-phase are detected. 
The crystalline phase of PVDF is essentially affected by the evaporation rate $[34,35]$. Low evaporation temperature until $90^{\circ} \mathrm{C}$ where crystallization is slow due to lower polymer-chain mobility, leads to preferential nucleation in the $\beta$-phase.

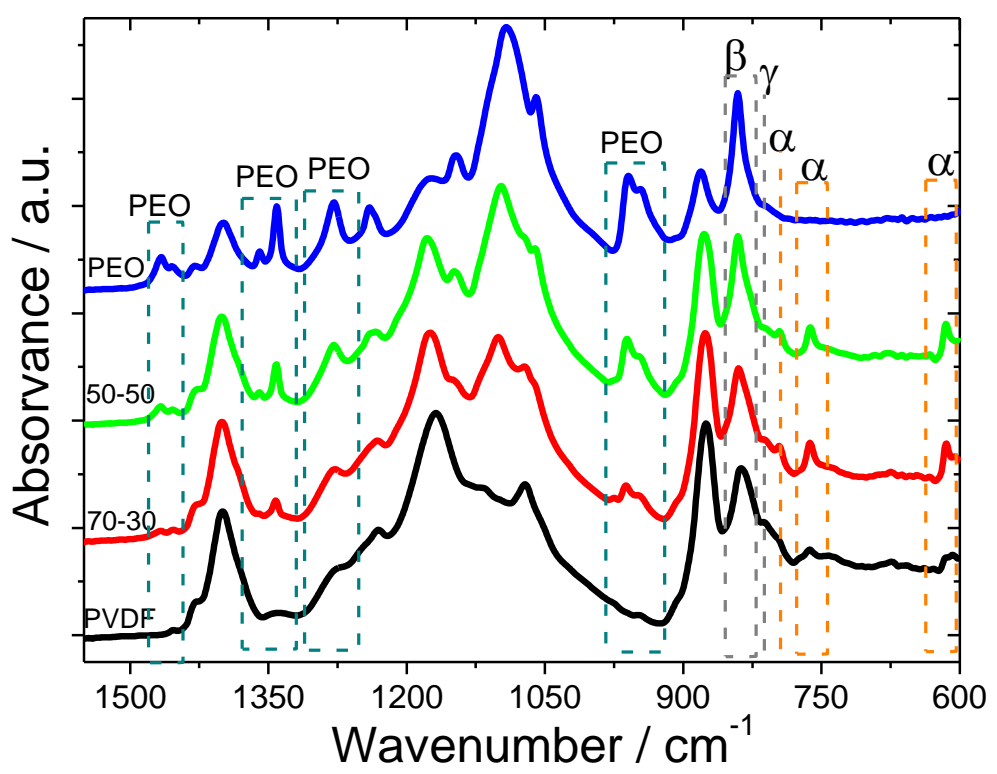

Figure 5 - FTIR-ATR spectra of the PVDF/PEO blends

In relation to PEO, the vibration bands at $948,1280,1343$ and $1468 \mathrm{~cm}^{-1}$ are observed, corresponding of $\mathrm{CH}_{2}$ rocking, twisting, wagging and scissoring, respectively. These specific bands of PEO are also detected in the PVDF/PEO blends and their intensity depends on the amount of PEO in the polymer blends.

The presence of PEO in the polymer blend modifies the intensity of the bands corresponding to PVDF, i.e, the vibration band of the $\alpha$-phase increase and the vibration band of $\beta$-phase decrease with increasing PEO content in the polymer blend.

The phase content of PVDF was calculated from the FTIR spectra through the method explained in [30]. The relative fraction of the $\beta$-phase in a sample containing $\alpha$ and $\beta$ PVDF is:

$$
F(\beta)=\frac{A_{\beta}}{\left(K_{\beta} / K_{\alpha}\right) A_{\alpha}+A_{\beta}}
$$

where $F(\beta)$, represents the $\beta$-phase content; $A_{\alpha}$ and $A_{\beta}$ the absorbance at 766 and 840 $\mathrm{cm}^{-1} ; \mathrm{K}_{\alpha}$ and $\mathrm{K}_{\beta}$ are the absorption coefficients at the respective wavenumber, which values are $6.1 \times 10^{4}$ and $7.7 \times 10^{4} \mathrm{~cm}^{2} \mathrm{~mol}^{-1}$, respectively. 
The quantification of $\beta$-phase of PVDF of the different polymer blends is shown in the table 1.

Table 1 - Thickness, $\beta$-phase content and piezoelectric coefficient of the PVDF/PEO polymer blends

\begin{tabular}{|c|c|c|c|}
\hline Samples & Thickness $/ \mu \mathbf{m}$ & $\mathbf{F}(\boldsymbol{\beta}) / \pm \mathbf{2 \%}$ & $\left|\mathbf{d}_{\mathbf{3 3}}\right| / \pm \mathbf{1} \mathbf{~ p C / N}$ \\
\hline PVDF & 81 & 85 & 5 \\
\hline PVDF/PEO (70/30) & 97 & 68 & 3 \\
\hline PVDF/PEO (50/50) & 120 & 74 & 2 \\
\hline PEO & 180 & ----- & ----- \\
\hline
\end{tabular}

Table 1 shows that the $\beta$-phase content decreases with the introduction of PEO, i.e, $85 \%$ of $\beta$-phase content is obtained for the PVDF pristine polymer in comparison with $74 \%$ of $\beta$-phase content for PVDF/PEO (50/50) or 68\% for PVDF/PEO (70/30). The difference of $\beta$-phase content between PVDF/PEO polymer blends is thus quite small and those variations does not have influence in the piezoelectric response [42]. A small peak indicating the presence of the $\gamma$-phase of PVDF has been detected also in the sample with 50\% PEO content, as indicated by the small band at $810 \mathrm{~cm}^{-1}$ [30].

The membranes thickness depends on the PEO polymer amount due to the difference in PVDF and PEO densities, its value varies between $81-180 \mu \mathrm{m}$ as shown in table 1 .

As the application of the membranes in tissue engineering shows the relevance of the piezoelectricity, that improves osteoblasts adhesion and proliferation [43], the piezoelectric response has been measured. Table 1 shows the overall piezoelectric coefficient $d_{33}$ of the samples. It is to notice that this value has to be interpreted taken into account that it represent the overall piezoelectric response of the sample, and not just the piezoelectric response of PVDF, i.e. variations due to blending are included. The modulus of the $d_{33}$ response of the PVDF/PEO polymer blend, fully ascribed to piezoelectric PVDF, is influenced by the relative PEO content, decreasing with increasing PEO content from $5 \mathrm{pC} / \mathrm{N}$ for pristine $\mathrm{PVDF}$ to 3 or $2 \mathrm{pC} / \mathrm{N}$ for the polymer blends with increasing PEO content from 30 to 50. Though the latter values are within experimental error, the sample with the largest PEO contents always provides the lower piezoelectric response. Independently of the polymer/blend type, the $d_{33}$ is negative and stable along the time. Comparing the $\mathrm{d}_{33}$ obtained of PVDF polymer in this work $\left(\left|\mathrm{d}_{33}\right|=\right.$ $5 \mathrm{pC} / \mathrm{N})$ and the $\mathrm{d}_{33}$ values typically obtained for PVDF films $\left(\left|\mathrm{d}_{33}\right|=20 \mathrm{pC} / \mathrm{N}\right)[42,44]$, 
the observed difference is attributed to the mechanical variations and the presence of some microporosity of the pristine PVDF obtained in this work. PVDF was obtained by evaporation at $70^{\circ} \mathrm{C}$ in order to follow the same protocol used for the blends with PEO and it has been shown that at this temperature pores appear in the sample due to solidliquid phase separation during solvent casting [31]. Porosity strongly affects microstructure and phase content of PVDF [31] and co-polymers [45], allowing tailoring those parameters for specific applications. It is to notice the relevance of obtaining piezoelectric response in the porous PVDF sample, scarcely addressed in the literature, and PVDF-PEO polymer blends, allowing implementation into technological applications, such as scaffolds and membranes, which involve the use of porous microstructures allied to piezoelectric sensing and actuation. Further, despite this decrease of the piezoelectric signal to respect to polymer films, the obtained electroactive response is still among the largest for piezoelectric polymers [28, 30], being within the range for applicability.

\subsection{Thermal properties}

In this section we analyze the behavior of PEO confined between PVDF domains. Since PVDF/PEO polymer blend films are obtained by solvent casting at $70^{\circ} \mathrm{C}$, PVDF crystals are formed while PEO is in the molten state so, its conformational mobility is high enough to reorganize while PVDF crystals grow and thus after solvent evaporation PEO occupies regions between the PVDF domains, and, as deduced from electron microscopy, within those domains as well. The study of the effect of the amount of PEO in the blend, and thus the size of the PEO regions after solvent evaporation, on its crystallization and melting processes and on the glass transition of amorphous regions informs about the degree of confinement of the PEO chains in the blend.

Figure 6 shows the DSC scans of neat PEO and PVDF/PEO blends for the cast samples at a heating rate of $20^{\circ} \mathrm{C} / \mathrm{min}$. Note that after film formation temperature did not overpass $70^{\circ} \mathrm{C}$, thus it is expected that PVDF domains remain unaltered in all thermal histories to what the sample is subjected. The first heating scan is representative of the melting of the crystal formed during cooling from $70^{\circ} \mathrm{C}$ to room temperature during blend formation process. The second scan was performed after cooling at $20^{\circ} \mathrm{C}$ after first scan in which PEO molten chains can rearrange in some extent. Heat flow has been normalized with the mass of PEO in the blend. It is worth note the important sift of the 
melting peak in 70/30 blend towards lower temperatures with respect to pure PEO. On the other hand the crystallization exothermal on cooling is also shifted to lower temperatures (Figure 7). It seems that crystallization kinetics is slower in the blend and thus, crystals are formed on cooling at lower temperatures. As a consequence, they are smaller and melt at lower temperatures as well. The behavior of 50/50 blend is in between neat PEO and 70/30 blend as can be seen clearly in the second scan. However, in the first scan it looks like in pure PEO, this means that PEO in 50/50 blend is susceptible to reorganize by melting and crystallization. It is worth note in support of this interpretation that there is nearly no difference between first and second heating scan in pure PEO but in the blends the melting peak in the second scan shifts to lower temperatures. These features could be confirmed by isothermal crystallization and further melting experiments

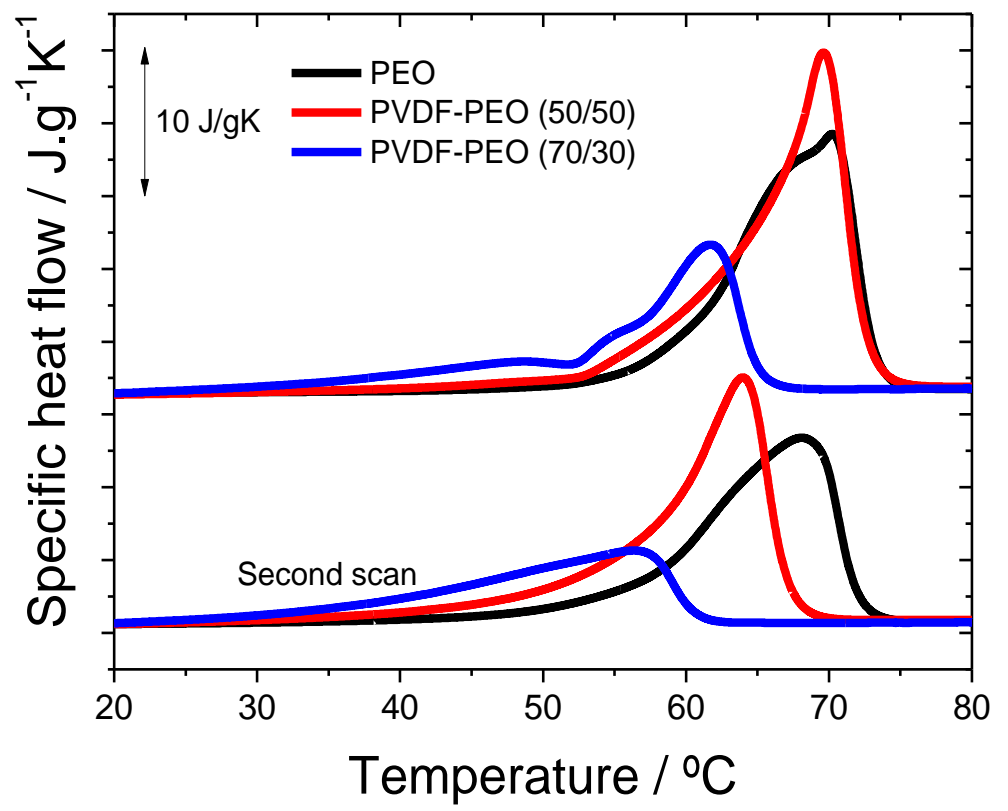

Figure 6 - Specific heat flow per gram of PEO vs temperature in DSC first and second scan for PEO polymer and PVDF-PEO blends.

Melting enthalpy for neat PEO, PVDF/PEO (50/50) and PVDF/PEO (70/30) are 170 $\mathrm{J} / \mathrm{g}, 90 \mathrm{~J} / \mathrm{g}$ ( $52 \%$ of neat PEO) and $32 \mathrm{~J} / \mathrm{g}$ (20\% of neat PEO), respectively, When calculating per gram of PEO, one finds that melting enthalpy in PVDF/PEO (50/50) sample is similar to that of neat PEO. It seems that PEO domains are large enough to allow PEO chains to diffuse and incorporate to growing crystals as in pure PEO. Nevertheless in PVDF/PEO (70/30) sample, the crystallinity attained in PEO phase is 
significantly smaller, so a fraction of PEO chains are dispersed in very small domains, perhaps between PVDF lamellae and are not able to crystallize. This fraction of dispersed PEO chains must be related with the fraction of PEO that is not dissolved when the sample is immersed in water.

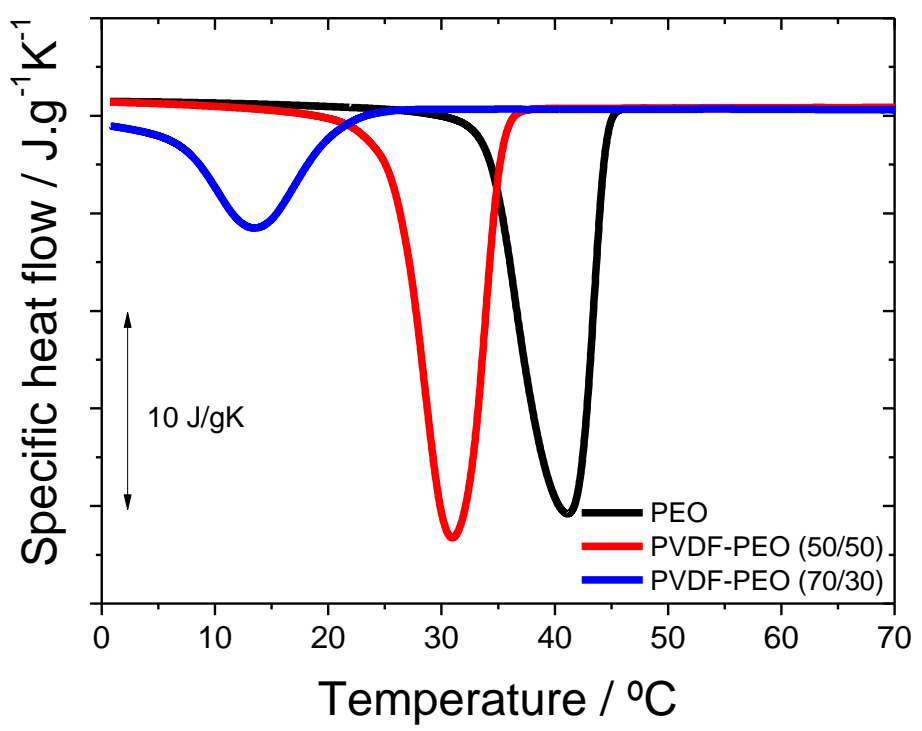

Figure 7 - Specific heat flow per gram of PEO vs temperature in DSC cool scan for PEO polymer and PVDF-PEO blends.

The differences found between the first and the second heating scans proves the ability of reorganizing PEO phase by successive melting and crystallization. This feature can be due to the miscibility of PVDF and PEO in amorphous phase. Crystallization kinetics can be influenced by the fact that PEO chains must separate from the homogeneous amorphous blend with PVDF chains and once they form the crystals and these crystals melts in the first scan, the homogeneous blend is not formed again because of the lack of time for spontaneous diffusion. To test this possibility the glass transition region was analyzed as shown in figure 8 for the two heating scans. 


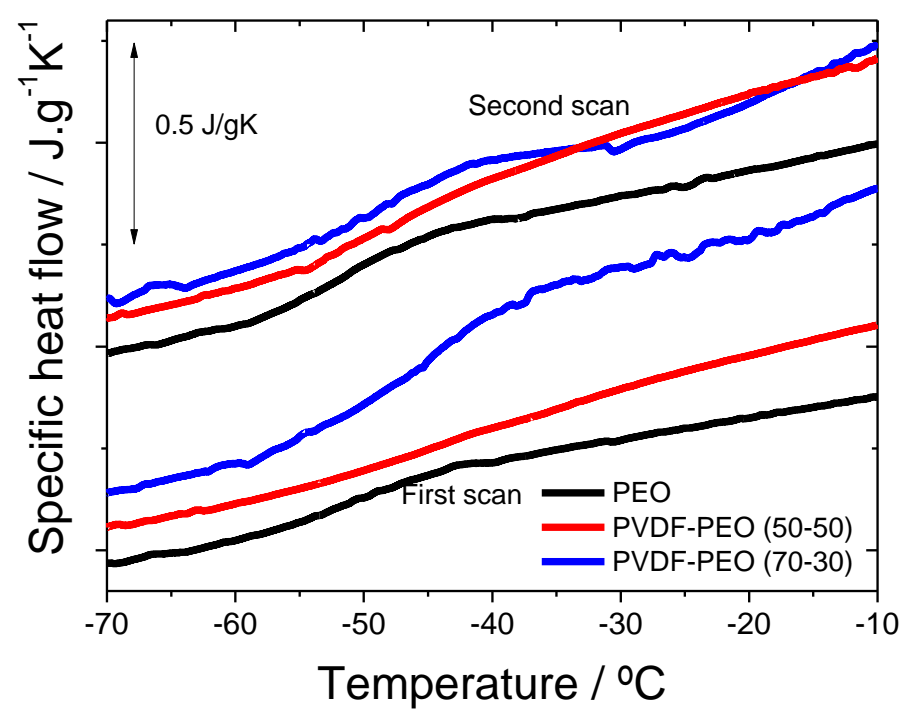

Figure 8 - Specific heat flow vs temperature in DSC first and second heating scan PEO polymer and PVDF-PEO blends.

Figure 8 shows the presence of the PEO glass transition region in the polymer blends at the same temperatures than for pure PEO, both in the first and in the second scan. Glass transition temperature was determined as the mid point of the rise of $\mathrm{C}_{\mathrm{p}}$ in the transition. The heat capacity increment at $\mathrm{T}_{\mathrm{g}}, \Delta \mathrm{C}_{\mathrm{p}}$, was determined per gram of PEO in the blend. The values of both parameters for all samples in the two heating scans are shown in table 2.

Table $2-\mathrm{T}_{\mathrm{g}}$ and $\Delta \mathrm{C}_{\mathrm{p}}$ referred to PEO content for all samples in the two heating scans.

\begin{tabular}{|l|c|c|c|c|}
\hline & $\begin{array}{c}\mathrm{T}_{\mathrm{g}}\left({ }^{\circ} \mathrm{C}\right) \\
1^{\text {st }} \mathrm{scan}\end{array}$ & $\begin{array}{c}\Delta \mathrm{Cp}(\mathrm{J} / \mathrm{gK}) \\
1^{\text {st }} \text { scan }\end{array}$ & $\begin{array}{c}\mathrm{T}_{\mathrm{g}}\left({ }^{\circ} \mathrm{C}\right) \\
2^{\text {nd }} \text { scan }\end{array}$ & $\begin{array}{c}\Delta \mathrm{Cp}(\mathrm{J} / \mathrm{gK}) \\
2^{\text {nd }} \text { scan }\end{array}$ \\
\hline Neat PEO & $-51 \pm 1$ & $0.10 \pm 0.02$ & $-50 \pm 1$ & $0.12 \pm 0.02$ \\
\hline PVDF/PEO (50/50) & $-48 \pm 1$ & $0.07 \pm 0.04$ & $-49 \pm 1$ & $0.11 \pm 0.04$ \\
\hline PVDF/PEO (70/30) & $-48 \pm 1$ & $0.20 \pm 0.06$ & $-50 \pm 1$ & $0.12 \pm 0.06$ \\
\hline
\end{tabular}

The shape of the glass transition in the first scan is quite different in the blends than in pure PEO but no clear trend with sample composition is shown. Nevertheless in the second scan both the glass transition temperature and the heat capacity increment in the transition is the same in all samples. It seems that the amorphous chains are constrained 
in blend during sample formation but melting during the first heating scan and recrystallization of PEO during cooling reorganize the amorphous phase that seems to behave similarly in the blends and the pure PEO

The different techniques applied to the characterization of PVDF/PEO blends show that the formation of PVDF phase by crystallization from the PVDF/PEO solution in DMF at $70^{\circ} \mathrm{C}$ depends on the amount of PEO in the solution. PVDF crystals are able to grow freely since PEO is molten in the whole process, but if the amount of PEO is large enough, it seems to create discontinuities in PVDF. In this work we have produced blends with 30 or $50 \%$ by weight of PEO but with intermediate compositions it seems that blend morphology could be modulated between the two different behaviors shown in this work. It seems that starting from a homogeneous solution of both components in the solvent, PVDF crystallization segregates an amorphous phase in which PEO and PVDF chains remain mixed. Then, on cooling, already without any solvent, PEO crystals are formed while confined by the previously formed PVDF crystals. This implies a further separation between PVDF and PEO chains. Reorganization of PEO phase by melting and crystallization is detected by the evolution of the glass transition and the melting process. The spatial continuity and close interaction in PEO-PVDF interphase is probed by the dependence of melting and crystallization kinetics of PEO with blend composition. The thermal treatment in the production of the blend film separates the crystallization processes of each component allowing a precise control of crystal growth. This technique could be extended to the manufacture of other polymer membranes.

\section{Conclusions}

PVDF/PEO blends were prepared with a procedure that allows confinement of PEO phase between quite homogeneous PVDF domains. This is done by crystallizing PVDF in a first stage, by casting from solution at a temperature at which PEO is molten. In a second stage PEO crystallizes on cooling, from the melt. The presence of PEO in the PVDF/PEO blends affects the crystallization process of PVDF. The vibration mode characteristics of PVDF are not influenced by the presence of PEO in the polymer blend but the $\beta$-phase content decreases with increasing the amount of the PEO polymer in the PVDF/PEO blends. Also the piezoelectric coefficient $\mathrm{d}_{33}$ of the PVDF/PEO blends depends on the amount of the PEO polymer in the PVDF/PEO blends. On the other 
hand large amounts of PEO in the solution makes that on solvent evaporation PVDF form non interconnected spherical aggregates. This fact is demonstrated by the collapse of PVDF phase when PEO is extracted from the blend film. On the contrary, when the amount of PEO is smaller, interconnexion between PVDF aggregates hinders in some extent membrane collapse on PEO extraction. PEO crystallization kinetics is largely affected by confinement between PVDF domains. Differences between first and second heating scans shows that some rearrangement of PEO domains takes place on crystallization and further melting, what together with the differences in glass transition temperature indicates a certain degree of miscibility between the amorphous phases of PEO and PVDF.

\section{Acknowledgements}

This work was funded by the Spanish Ministry of Economy and Copetitiveness (MINECO) through the project MAT2013-46467-C4-1-R (including the FEDER financial support) and by FEDER funds through the "Programa Operacional Factores de Competitividade e COMPETE" and by national funds from FCT e Fundação para a Ciência e a Tecnologia, in the framework of the strategic project Strategic Project PEST -C/FIS/UI607/2014. The authors also thank funding from Matepro - Optimizing Materials and Processes", ref.NORTE-07-0124-FEDER-000037”, co-funded by the "Programa Operacional Regional do Norte" (ON.2 e O Novo Norte), under the "Quadro de Referência Estratégico Nacional" (QREN), through the "Fundo Europeu de 
Desenvolvimento Regional" (FEDER), and grant SFRH/BD/68499/2010 (C.M.C.). CIBER-BBN is an initiative funded by the VI National R\&D\&i Plan 2008-2011, Iniciativa Ingenio 2010, Consolider Program, CIBER Actions and financed by the Instituto de Salud Carlos III with assistance from the European Regional Development Fund. MNTM acknowledges the Programa de Ayuda de Investigación y Desarrollo (PAID) of the Universitat Politècnica de València for her doctoral grant. The authors acknowledge the assistance and advice of Electron Microscopy Service of the UPV

\section{References}

[1] Wen Q, Di J, Zhao Y, Wang Y, Jiang L, Yu J. Flexible inorganic nanofibrous membranes with hierarchical porosity for efficient water purification. Chemical Science. 2013;4:4378-82.

[2] Košutić K, Kaštelan-Kunst L, Kunst B. Porosity of some commercial reverse osmosis and nanofiltration polyamide thin-film composite membranes. Journal of Membrane Science. 2000;168:101-8.

[3] Adiga SP, Jin C, Curtiss LA, Monteiro-Riviere NA, Narayan RJ. Nanoporous membranes for medical and biological applications. Wiley Interdisciplinary Reviews: Nanomedicine and Nanobiotechnology. 2009;1:568-81.

[4] Ting CL, Appelö D, Wang Z-G. Minimum Energy Path to Membrane Pore Formation and Rupture. Physical Review Letters. 2011;106:168101.

[5] Zhang J, Sun B, Huang X, Chen S, Wang G. Honeycomb-like porous gel polymer electrolyte membrane for lithium ion batteries with enhanced safety. Sci Rep. 2014;4. 
[6] Li Y, Fu Z-Y, Su B-L. Hierarchically Structured Porous Materials for Energy Conversion and Storage. Advanced Functional Materials. 2012;22:4634-67.

[7] Yang Q, Adrus N, Tomicki F, Ulbricht M. Composites of functional polymeric hydrogels and porous membranes. Journal of Materials Chemistry. 2011;21:2783-811.

[8] Wang LK, Chen JP, Hung YT, Shammas NK. Membrane and Desalination Technologies: Humana Press; 2010.

[9] Mulder M. Basic Principles of Membrane Technology: Kluwer Academic; 1996.

[10] Matsuura T. Synthetic Membranes and Membrane Separation Processes: CRC Press; 1994.

[11] Li NN, Fane AG, Ho WSW, Matsuura T. Advanced Membrane Technology and Applications: John Wiley \& Sons; 2008.

[12] Guillen GR, Pan Y, Li M, Hoek EMV. Preparation and Characterization of Membranes Formed by Nonsolvent Induced Phase Separation: A Review. Industrial \& Engineering Chemistry Research. 2011;50:3798-817.

[13] Olson F, Hunt CA, Szoka FC, Vail WJ, Papahadjopoulos D. Preparation of liposomes of defined size distribution by extrusion through polycarbonate membranes. Biochimica et Biophysica Acta (BBA) - Biomembranes. 1979;557:9-23.

[14] Wang D, Li K, Teo WK. Porous PVDF asymmetric hollow fiber membranes prepared with the use of small molecular additives. Journal of Membrane Science. 2000;178:13-23.

[15] Kitamura T, Okabe S, Tanigaki M, Kurumada K-I, Ohshima M, Kanazawa S-I. Morphology change in polytetrafluoroethylene (PTFE), porous membrane caused by heat treatment. Polymer Engineering \& Science. 2000;40:809-17.

[16] Matsuyama H, Maki T, Teramoto M, Asano K. Effect of polypropylene molecular weight on porous membrane formation by thermally induced phase separation. Journal of Membrane Science. 2002;204:323-8.

[17] Ghosh AK, Hoek EMV. Impacts of support membrane structure and chemistry on polyamide-polysulfone interfacial composite membranes. Journal of Membrane Science. 2009;336:140-8.

[18] Rambo CR, Recouvreux DOS, Carminatti CA, Pitlovanciv AK, Antônio RV, Porto LM. Template assisted synthesis of porous nanofibrous cellulose membranes for tissue engineering. Materials Science and Engineering: C. 2008;28:549-54.

[19] Nalwa HS. Ferroelectric Polymers: Chemistry, Physics, and Applications: M. Dekker, Incorporated; 1995. 
[20] Rasmussen L. Electroactivity in Polymeric Materials: Springer; 2012.

[21] Zhu L-P, Yu J-Z, Xu Y-Y, Xi Z-Y, Zhu B-K. Surface modification of PVDF porous membranes via poly(DOPA) coating and heparin immobilization. Colloids and Surfaces B: Biointerfaces. 2009;69:152-5.

[22] Chang Y, Shih Y-J, Ruaan R-C, Higuchi A, Chen W-Y, Lai J-Y. Preparation of poly(vinylidene fluoride) microfiltration membrane with uniform surfacecopolymerized poly(ethylene glycol) methacrylate and improvement of blood compatibility. Journal of Membrane Science. 2008;309:165-74.

[23] Boschin F, Blanchemain N, Bria M, Delcourt-Debruyne E, Morcellet M, Hildebrand HF, et al. Improved drug delivery properties of PVDF membranes functionalized with $\beta$-cyclodextrin-Application to guided tissue regeneration in periodontology. Journal of Biomedical Materials Research Part A. 2006;79A:78-85.

[24] Li H, Chen Y-M, Ma X-T, Shi J-L, Zhu B-K, Zhu L-P. Gel polymer electrolytes based on active PVDF separator for lithium ion battery. I: Preparation and property of PVDF/poly(dimethylsiloxane) blending membrane. Journal of Membrane Science. 2011;379:397-402.

[25] Costa CM, Silva MM, Lanceros-Mendez S. Battery separators based on vinylidene fluoride (VDF) polymers and copolymers for lithium ion battery applications. RSC Advances. 2013;3:11404-17.

[26] Yang X, Zhang B, Liu Z, Deng B, Yu M, Li L, et al. Preparation of the antifouling microfiltration membranes from poly(N,N-dimethylacrylamide) grafted poly(vinylidene fluoride) (PVDF) powder. Journal of Materials Chemistry. 2011;21:11908-15.

[27] Madaeni SS, Taheri AH. Effect of Casting Solution on Morphology and Performance of PVDF Microfiltration Membranes. Chemical Engineering \& Technology. 2011;34:1328-34.

[28] Lovinger AJ. Developments in Crystalline Polymers. In: Basset DC, editor. London: Elsevier; 1982.

[29] Gregorio JR, Cestari M. Effect of crystallization temperature on the crystalline phase content and morphology of poly(vinylidene fluoride). Journal of Polymer Science Part B: Polymer Physics. 1994;32:859-70.

[30] Martins P, Lopes AC, Lanceros-Mendez S. Electroactive phases of poly(vinylidene fluoride): Determination, processing and applications. Progress in Polymer Science. 2014;39:683-706. 
[31] Magalhães R, Durães N, Silva M, Silva J, Sencadas V, Botelho G, et al. The Role of Solvent Evaporation in the Microstructure of Electroactive $\beta$-Poly(Vinylidene Fluoride) Membranes Obtained by Isothermal Crystallization. Soft Materials. 2010;9:114.

[32] Su Y, Chen C, Li Y, Li J. PVDF Membrane Formation via Thermally Induced Phase Separation. Journal of Macromolecular Science, Part A. 2007;44:99-104.

[33] Cui Z-Y, Xu Y-Y, Zhu L-P, Wang J-Y, Zhu B-K. Investigation on PVDF-HFP microporous membranes prepared by TIPS process and their application as polymer electrolytes for lithium ion batteries. Ionics. 2009;15:469-76.

[34] Chinaglia DL, Gregorio R, Stefanello JC, Pisani Altafim RA, Wirges W, Wang F, et al. Influence of the solvent evaporation rate on the crystalline phases of solution-cast poly(vinylidene fluoride) films. Journal of Applied Polymer Science. 2010;116:785-91.

[35] Gregorio R, Ueno EM. Effect of crystalline phase, orientation and temperature on the dielectric properties of poly (vinylidene fluoride) (PVDF). Journal of Materials Science. 1999;34:4489-500.

[36] Ribeiro C, Moreira S, Correia V, Sencadas V, Rocha JG, Gama FM, et al. Enhanced proliferation of pre-osteoblastic cells by dynamic piezoelectric stimulation. RSC Advances. 2012;2:11504-9.

[37] Pärssinen J, Hammarén H, Rahikainen R, Sencadas V, Ribeiro C, Vanhatupa S, et al. Enhancement of adhesion and promotion of osteogenic differentiation of human adipose stem cells by poled electroactive poly(vinylidene fluoride). Journal of Biomedical Materials Research Part A. 2015;103:919-28.

[38] Burdick JA, Mauck RL. Biomaterials for Tissue Engineering Applications: A Review of the Past and Future Trends: Springer; 2010.

[39] Xi J, Qiu X, Li J, Tang X, Zhu W, Chen L. PVDF-PEO blends based microporous polymer electrolyte: Effect of PEO on pore configurations and ionic conductivity. Journal of Power Sources. 2006;157:501-6.

[40] Xiao Q, Wang X, Li W, Li Z, Zhang T, Zhang H. Macroporous polymer electrolytes based on PVDF/PEO-b-PMMA block copolymer blends for rechargeable lithium ion battery. Journal of Membrane Science. 2009;334:117-22.

[41] Costa CM, Machiavello MNT, Ribelles JLG, Lanceros-Méndez S. Compositiondependent physical properties of poly[(vinylidene fluoride)-co-trifluoroethylene]poly(ethylene oxide) blends. Journal of Materials Science. 2013;48:3494-504. 
[42] Gomes J, Nunes JS, Sencadas V, Lanceros-Mendez S. Influence of the $\beta$-phase content and degree of crystallinity on the piezo- and ferroelectric properties of poly(vinylidene fluoride). Smart Materials and Structures. 2010;19:065010.

[43] Martins PM, Ribeiro S, Ribeiro C, Sencadas V, Gomes AC, Gama FM, et al. Effect of poling state and morphology of piezoelectric poly(vinylidene fluoride) membranes for skeletal muscle tissue engineering. RSC Advances. 2013;3:17938-44.

[44] Silva MP, Costa CM, Sencadas V, Paleo AJ, Lanceros-Méndez S. Degradation of the dielectric and piezoelectric response of $\beta$-poly(vinylidene fluoride) after temperature annealing. J Polym Res. 2011;18:1451-7.

[45] California A, Cardoso VF, Costa CM, Sencadas V, Botelho G, Gómez-Ribelles JL, et al. Tailoring porous structure of ferroelectric poly(vinylidene fluoridetrifluoroethylene) by controlling solvent/polymer ratio and solvent evaporation rate. European Polymer Journal. 2011;47:2442-50. 